\title{
Benzodiazépines : vous avez dit concentrations mortelles...
}

\author{
Benzodiazepines : \\ fatal concentrations isn't it?
}

\section{Jean-Pierre GOULLE' ${ }^{*(1)}$, Jean NOUVEAU ${ }^{(2)}$, Patricia BRIQUET ${ }^{(3)}$, Hervé DEBRIS ${ }^{(4)}$, Vincent CIRIMELE ${ }^{(5)}$, Pascal KINTZ ${ }^{(5)}$}

(1) Laboratoire de Pharmacocinétique et de Toxicologie Clinique - Groupe Hospitalier B.P. 24 - 76083 LE HAVRE Cedex - Tél : 0232733218 - Fax : 0232733238

(2) Service de Réanimation Médicale - Groupe Hospitalier B.P. 24 - 76083 LE HAVRE Cedex - Tél : 0232733190 - Fax : 0232733241

(3) Département Accueil du Traitement des Urgences - Groupe Hospitalier B.P. 24 - 76083 LE HAVRE Cedex - Tél : 0232733163 - Fax : 0232733412

(4) Service d'Accueil Médical d'Urgence - Groupe Hospitalier

B.P. 24 - 76083 LE HAVRE Cedex - Tél : 0232733131

(5) Institut de Médecine Légale, 11, rue Humann - 67000 STRASBOURG Tél : 0390243347 - Fax : 0390243362

* Auteur à qui adresser la correspondance : Dr Jean-Pierre GOULLÉ, Laboratoire de Pharmacocinétique et de Toxicologie Cliniques - Groupe Hospitalier Jacques Monod - B.P. 24 - 76083 LE HAVRE Tél : 0232733223 - Fax : 0232733238 - e-mail : jgoulle@ch-havre.fr

(Reçu le 26 avril 2001 ; accepté le 21 mai 2001)

\section{$R E ́ S U M E ́$}

L'objectif de ce travail est de présenter deux cas d'ingestion massive de benzodiazépines (BZD) et de montrer l'extraordinaire tolérance de sujets accoutumés à la consommation de ces molécules. La première observation concerne un homme de 44 ans, en invalidité pour dépression, traité par venlafaxine et clorazépate qui ingère, suite à un différent familial 34,5 g de clorazépate. A l'arrivée aux urgences, le malade est somnolent mais réveillable. Il est admis par sécurité en réanimation malgré un score de Glasgow à 15 . Il est somnolent pendant 3 jours. Il est ensuite transféré en médecine puis en psychiatrie et quitte l'hôpital le $12^{\text {eme }}$ jour. Onze prélèvements sanguins sont réalisés ainsi qu'un prélèvement de cheveux. La seconde observation est celle d'une femme de 38 ans, ancienne toxicomane par voie veineuse, VIH I positive, qui consulte dans le cadre du traitement de son SIDA. En raison de troubles de la vigilance ainsi que de l'élocution, le médecin demande un bilan toxicologique sanguin et urinaire, orienté vers la recherche de stupéfiants. Des che-

\section{SUMMARY}

The purpose of this paper is to present two cases of severe intoxication with benzodiazepines (BZD) and the extraordinary individual tolerance to these compounds. The first case is that of a 44-year-old man, having a bad fit of depression, treated with venlafaxine and clorazepate who ingested $34.5 \mathrm{~g}$ of clorazepate after a domestic quarrel. When admitted to the hospital, the patient was drowsy without coma. Despite a Glasgow's score of 15, he was admitted to the emergency unit. There, he remained sleepy for 3 days. Then he was admitted in a medical unit, later in a psychiatric unit and left the hospital after 12 days. Eleven blood samples, as well hair samples, were collected. The second case concerns a 38-year-old woman, addicted in the past to heroin, HIV I positive, who consulted for AIDS treatment. The physician collected blood, urine and hair for toxicological screening (especially drugs of abuse), because of wording and balance disorders. In both cases, BZD were assayed by HPLC/DAD in blood and by GC/MS using negative chemical ionization 
veux sont prélevés. Dans les deux cas, l'identification et le dosage des BZD plasmatiques sont réalisés par CLUV/BD et le dosage des BZD dans les cheveux par CG/SM en mode ionisation chimique négative. Pour la première observation les concentrations sanguines varient sur la période de 12 jours entre 54 et $19 \mathrm{mg} / \mathrm{l}$ pour le nordazépam et de 6,53 à $0,35 \mathrm{mg} / \mathrm{l}$ pour l'oxazépam. Les dosages dans les cheveux montrent des concentrations respectives de 6,23 et 0,95 ng/mg. Quant à la seconde observation, les dosages sanguins et capillaires permettent de mettre en évidence une conduite toxicophile aux BZD : nordazépam 31,7 $\mathrm{mg} / \mathrm{l}$ et $5,77 \mathrm{ng} / \mathrm{mg}$; diazépam $8,2 \mathrm{mg} / \mathrm{l}$ et $3,02 \mathrm{ng} / \mathrm{mg}$; oxazépam $3,2 \mathrm{mg} / \mathrm{l}$ et $0,67 \mathrm{ng} / \mathrm{mg}$; lorazépam $0,26 \mathrm{mg} / \mathrm{l}$ et $0,26 \mathrm{ng} / \mathrm{mg}$; lormétazépam 0,18 $\mathrm{mg} / \mathrm{l}$ et $0,39 \mathrm{ng} / \mathrm{mg}$. Ces deux observations mettent en évidence des phénomènes d'accoutumance et de tolérance chez des sujets présentant des conduites toxicophiles anciennes. Elles contrastent avec les cas décrits dans la littérature de sujets décédés d'intoxications mettant en cause uniquement une BZD, mais également avec les cas tirés de notre expérience médico-légale, pour lesquels les concentrations mesurées, admises comme étant à l'origine du décès, sont souvent nettement inférieures à celles mesurées chez ces deux malades.

\section{MOTS-CLÉS}

Intoxication, benzodiazépines, sang, cheveux.

\section{Introduction}

Les benzodiazépines figurent parmi les médicaments les plus utilisés en France. Si la prescription médicale initiale a pour objectif d'assurer une prise en charge optimale des malades, la survenue d'intoxications aiguës volontaires aux benzodiazépines est fréquente et bien documentée (1). Dans la série strasbourgeoise portant sur 4078 cas d'intoxications par benzodiazépines observées dans un service de réanimation et urgences médicales, $75 \%$ des patients avaient pris au moins une benzodiazépine. Il s'agit dans l'immense majorité d'intoxications ne nécessitant aucun traitement particulier mais une simple surveillance, généralement de courte durée. Leur pronostic est bon, la mortalité en milieu hospitalier est voisine de $0,1 \%$ (1). En revanche, en l'absence de prise en charge médicale, le nombre de décès répertoriés est beaucoup plus important $(0,68 \%$ versus $0,11 \%$ ), comme le montre une étude réalisée dans le nord de la France (2). Outre les intoxications aiguës volontaires aux benzodiazépines, les conduites addictives à ces molécules sont bien connues mais elles étonnent toujours par les quantités considérables que certains sujets sont capables d'ingérer. L'extraordinaire tolérance clinique constatée ne peut s'expliquer que par l'accoutumance à ce qui n'est plus de la thérapeutique mais de la pharmacodépendance. Nous rapportons deux cas de conduite toxicophile aux benzodiazépines documentés par l'analyse du sang et des cheveux. in hair. In the first case, blood concentrations decreased over the 12 day-period from 54 to $19 \mathrm{mg} / \mathrm{l}$ for nordazepam and from 6.53 to $0.35 \mathrm{mg} / \mathrm{l}$ for oxazepam. The respective hair concentrations were 6.23 and $0.95 \mathrm{ng} / \mathrm{mg}$. In the second case, blood and hair determination demonstrated the addiction to $B Z D$ : nordazepam $31.7 \mathrm{mg} / \mathrm{l}$ and $5.77 \mathrm{ng} / \mathrm{mg}$; diazepam $8.2 \mathrm{mg} / \mathrm{l}$ and $3.02 \mathrm{ng} / \mathrm{mg}$; oxazepam $3.2 \mathrm{mg} / \mathrm{l}$ and 0.67 $\mathrm{ng} / \mathrm{mg}$; lorazepam $0.26 \mathrm{mg} / \mathrm{l}$ and $0.26 \mathrm{ng} / \mathrm{mg}$; lormetazepam 0.18 and $0.39 \mathrm{ng} / \mathrm{mg}$. These results clearly document the extraordinary tolerance to BZD in subjects exposed to these drugs. These cases contrast with those previously published concerning subjects who died from single BZD poisoning, but also with our forensic cases, in which so-called 'fatal' blood concentrations are often lower than those measured in these two patients.

\section{KEY-WORDS}

Intoxication, benzodiazepines, blood, hair analysis.

L'une des observations se double d'une tentative de suicide au médicament, objet de la conduite addictive.

\section{Matériel et méthode}

\section{Recherche des stupéfiants dans les urines}

La recherche est effectuée par méthode d'immunopolarisation de fluorescence (FPIA) sur automate AXSYM Abbott (Rungis, France) à l'aide des réactifs destinés à cet appareil.

\section{Recherche et dosage des benzodiazé- pines plasmatiques par chromatogra- phie liquide haute performance couplée à une barrette de diodes (CLHP-BD) Réactifs}

Les solvants sont de qualité CLHP, les autres produits chimiques sont de qualité pour analyse (Prolabo, Chelles, France). En ce qui concerne les benzodiazépines, les produits purs proviennent des laboratoires pharmaceutiques commercialisant les différentes spécialités.

\section{Préparation des échantillons et méthode de dosa-} ge des benzodiazépines

Le dosage des cinq benzodiazépines (desméthyldiazépam, diazépam, oxazépam, lorazépam et lormétazépam) est réalisé par chromatographie liquide haute per- 
formance couplée à une barrette de diodes (HPLC$\mathrm{BD})$. Après extraction de $1 \mathrm{ml}$ de plasma en présence de prazépam (étalon interne) en milieu alcalin $(180 \mu \mathrm{l}$ $\mathrm{Na} \mathrm{OH} 0,1 \mathrm{M}$ ) avec $2 \mathrm{ml}$ d'un mélange dichlorométhane/ hexane/acétate d'éthyle (5/4/1, v/v) et évaporation à sec de la phase organique, l'extrait obtenu est repris par du méthanol. $20 \mu \mathrm{l}$ sont ensuite injectés dans le système chromatographique Hewlett Packard (Les Ulis, France) série 1050 équipé d'un détecteur 1040 série II et d'une station de traitement de données Vectra. La phase mobile est constituée d'un gradient de concentration d'un mélange acétonitrile/tampon phosphate $(\mathrm{pH} 7)$. Les spectres UV sont enregistrés en continu de 200 à $400 \mathrm{~nm}$ (temps de rétention et longueur d'onde d'absorption maximum : diazépam 18,0 $\min -\lambda$ max $230 \mathrm{~nm}$; nordazépam $15,9 \mathrm{~min}-\lambda \max 228 \mathrm{~nm}$; oxazépam 14,8 min - $\lambda$ max $230 \mathrm{~nm}$; lorazépam 13,9 min - $\lambda$ max $228 \mathrm{~nm}$; lormétazépam 16,4 min - $\lambda$ max 232 $\mathrm{nm}$ ) et comparés à ceux de la bibliothèque. Pour l'analyse quantitative, une gamme étalon (diazépam, nordazépam et oxazépam : $0-1-5-10 \mathrm{mg} / 1$; lorazépam et lormétazépam : $0-0,1-0,2-0,5 \mathrm{mg} / \mathrm{l}$ ) est traitée dans les même conditions que l'échantillon biologique. Le prazépam est utilisé comme étalon interne.

\section{Dosage des benzodiazépines dans les cheveux par chromatographie gazeuse couplée à un détecteur de masse en mode d'ionisation chimique négative (CG- SM/ICN)}

\section{Réactifs}

Les solvants sont de qualité CLHP, les autres produits chimiques de qualité pour analyses (Merck, Dermstadt, Allemagne). Les benzodiazépines et leurs homologues deutérés sont de marque Radian (Promochem, Molsheim, France). Le N,O-Bis (triméthylsilyl) trifluoroacétamide (BSTFA) $+1 \%$ de triméthylchlorosilane (TMCS) provient de chez Interchim (Montluçon, France).

\section{Préparation des échantillons}

Les cheveux sont décontaminés par deux lavages de 2 min. chacun dans $5 \mathrm{ml}$ de dichlorométhane, à température ambiante. Ils sont ensuite pulvérisés dans un broyeur à boulet de marque Retsch type MM2 (Haan, Allemagne). Chaque prise d'essai, voisine de $50 \mathrm{mg}$, est incubée 20 heures à $40^{\circ} \mathrm{C}$ dans un tampon phosphate Soerensen, $\mathrm{pH} 7,6\left(38,8 \mathrm{ml}\right.$ de $\mathrm{KH}_{2} \mathrm{PO}_{4}$ à $9,07 \mathrm{~g} / 1$ et $61,2 \mathrm{ml}$ de $\mathrm{Na}_{2} \mathrm{HPO}_{4}$ à $\left.11,87 \mathrm{~g} / \mathrm{l}\right)$ en présence de $200 \mathrm{ng}$ de chaque étalon interne deutéré. L'homogénat est directement extrait par $5 \mathrm{ml}$ d'un mélange diéthy- léther-chloroforme $(80: 20, \mathrm{v} / \mathrm{v})$. Après $10 \mathrm{~min}$. d'agitation et $10 \mathrm{~min}$. de centrifugation à $2000 \mathrm{~g}$, la phase organique recueillie est évaporée à siccité. Le résidu est dérivé par silylation avec $35 \mu \mathrm{l}$ de BSTFA $+1 \%$ de TMCS pendant $20 \mathrm{~min}$. à $90^{\circ} \mathrm{C}$. Après dérivation, $1 \mu \mathrm{l}$ de l'extrait est injecté à l'aide d'un injecteur automatique HP 7673 dans la colonne de chromatographie.

\section{Méthode de CG-SM/ICN}

L'ensemble de CG-SM est constitué d'un chromatographe Hewlett Packard (5890) équipé d'un détecteur de masse (Engine 5989 B) avec une source chauffée à $200^{\circ} \mathrm{C}$. La tension du multiplicateur d'électrons est réglée pour les mesures à $+400 \mathrm{~V}$ au dessus de la valeur d'autotune en ICN. Le débit du gaz vecteur (hélium de pureté N55) dans la colonne est de $1 \mathrm{ml} / \mathrm{min}$. (colonne capillaire HP-5 MS, $5 \%$ de phényl et $95 \%$ de méthylsiloxane, $30 \mathrm{~m} \times 0,25 \mathrm{~mm}$ de diamètre interne et film de $0,25 \mu \mathrm{m}$ d'épaisseur). La température de l'injecteur est de $250^{\circ} \mathrm{C}$ et l'injection est réalisée en mode splitless avec un temps de fermeture de vanne de $0,75 \mathrm{~min}$. La température de la colonne est maintenue $1 \mathrm{~min}$. à $60^{\circ} \mathrm{C}$ puis portée à $290^{\circ} \mathrm{C}$ à raison de $30^{\circ} \mathrm{C} / \mathrm{min}$., température à laquelle elle est maintenue $2 \mathrm{~min}$. Les acquisitions sont réalisées en mode courant ionique total de 170 à 450 uma. Le méthane à la pression de $0,2 \mathrm{kPa}$ est utilisé comme gaz de réaction dans la source. Les benzodiazépines sont identifiées et quantifiées à l'aide des étalons deutérés par comparaison des temps de rétention et de l'aire des ions de quantification avec l'aide de deux ions de confirmation. Les courbes de calibration sont obtenues par addition de $5(0,1 \mathrm{ng} / \mathrm{mg}), 25(0,5$ $\mathrm{ng} / \mathrm{mg}), 50(1,0 \mathrm{ng} / \mathrm{mg}), 100(2,0 \mathrm{ng} / \mathrm{mg}), 500(10,0$ $\mathrm{ng} / \mathrm{mg})$ et $1000(20,0 \mathrm{ng} / \mathrm{mg}) \mathrm{ng}$ d'étalons purs préparés dans le méthanol à $50 \mathrm{mg}$ de cheveux témoins pulvérisés exempts de benzodiazépine. Les performances détaillées de cette technique ont été publiées (3).

\section{Observations}

\section{Observation 1}

Il s'agit d'un homme de 44 ans, en invalidité pour syndrome dépressif sévère depuis 4 ans. Dans les antécédents, on note un éthylisme sevré depuis 8 ans, sans rechute. Ce malade est traité par venlafaxine (Effexor $\left.{ }^{\circledR}\right)$ et par clorazépate (Tranxène $50^{\circledR}$ ). Il est supposé avoir absorbé, à la suite d'un différent familial, 23 boites de Tranxène $50^{\circledR}$ soit $34,5 \mathrm{~g}$ de clorazépate. Il est somnolent à l'arrivée aux urgences. Un lavage gastrique est tenté 1 heure 30 minutes après l'ingestion compte tenu de l'absorption massive. Celui-ci ramène des comprimés en début de lavage. Malgré un score de Glasgow à 15 , le malade est admis en réanimation, par sécurité 
mais aussi en raison d'une concentration plasmatique extraordinairement élevée $(49 \mathrm{mg} / \mathrm{l})$ obtenue par dosage semi-quantitatif de benzodiazépines, réalisé en garde par immunoanalyse. Là, on ne constate aucune complication si ce n'est une période de rétention d'urines de 12 heures pour laquelle une sonde est mise en place. Il reste somnolent pendant 3 jours mais ne reçoit aucun traitement antidotique. Il est ensuite transféré en médecine puis en psychiatrie d'où il sort le $12^{\text {eme }}$ jour. Des prélèvements sanguins, ainsi qu'un prélèvement de cheveux sont réalisés pendant l'hospitalisation. Les résultats des dosages par CLHP/UV/BD dans le sang et par CG-SM/ICN dans les cheveux mettent en évidence des concentrations très importantes regroupées dans la figure 1.

\section{Observation 1}

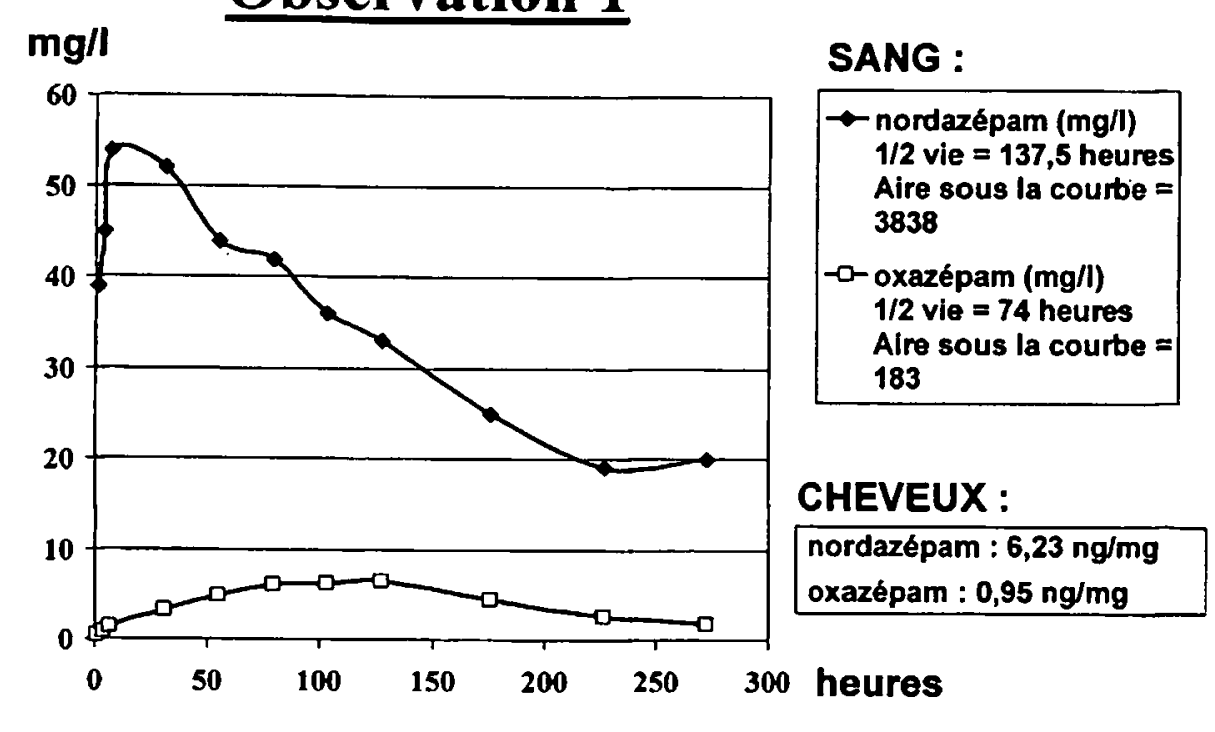

Figure 1 : Observation 1 , évolution des concentrations plasmatiques et des constantes toxicocinétiques en nordazépam et en oxazépam après intoxication aiguë volontaire au clorazépate. Concentrations en nordazépam et en oxazépam dans les cheveux.

\section{Observation 2}

Elle concerne une femme de 38 ans, VIH 1 positive à la suite d'une toxicomanie par voie intraveineuse. Elle est amenée à consulter pour le traitement de son SIDA. Le praticien constate des troubles de la vigilance et de l'élocution confirmés par l'interrogatoire, l'intéressée avouant des chutes à répétition responsables d'ecchymoses multiples. Craignant une rechute de sa conduite toxicophile, il réalise immédiatement un bilan toxicologique sanguin, urinaire et prélève des cheveux. La recherche des stupéfiants dans les urines est négative. Le dépistage sanguin par CLHP/BD met en évidence la présence de 5 benzodiazépines. Les dosages révèlent des concentrations considérables de benzodiazépines dans le sang et les cheveux reportées dans le tableau I.

\section{Discussion}

\section{Observation 1}

Monsieur F... dort toute la journée, cela est très bien décrit par lui-même et par son entourage ; il ne se lève que pour aller conduire ou chercher ses enfants à l'école. Il pratique manifestement le nomadisme médical, ce qui lui permet de consommer de grandes quantités de Tranxène $50^{\circledR}$ et de disposer de stocks considérables. Cette conduite toxicophile cachée est objectivée par l'extraordinaire tolérance clinique malgré des concentrations sanguines majeures, respectivement 30 fois et 10 fois la concentration considérée comme "toxique", à la phase aiguë de son intoxication puis à la sortie de l'hôpital 12 jours plus tard. Les dosages réalisés dans le sang et les cheveux permettent de valider l'interrogatoire et d'affirmer que l'intéressé a remplacé sa dépendance à l'alcool par une toxicomanie au clorazépate.

Tableau I : Observation 2, concentrations des cinq benzodiazépines mises en évidence dans le plasma et les cheveux.

\begin{tabular}{|l|c|c|c|c|}
\hline & $\begin{array}{c}\text { Plasma } \\
\text { (valeurs usuelles) } \\
\mathrm{mg} / 1\end{array}$ & $\begin{array}{c}0 \rightarrow 3 \mathrm{~cm} \\
(45 \mathrm{mg})\end{array}$ & $\begin{array}{c}3 \rightarrow 6 \mathrm{~cm} \\
(33 \mathrm{mg})\end{array}$ & $\begin{array}{c}6 \rightarrow 9 \mathrm{~cm} \\
(38 \mathrm{mg})\end{array}$ \\
\cline { 2 - 5 } & $\begin{array}{c}31,7 \\
(0,5-1,2)\end{array}$ & 5,77 & 3,12 & 2,39 \\
\hline Dordazépam & $\begin{array}{c}8,2 \\
(0,125-0,75)\end{array}$ & 3,02 & 1,20 & 0,98 \\
\hline Oxazépam & $\begin{array}{c}3,2 \\
(0,2-2,0)\end{array}$ & 0,67 & 0,32 & 0,15 \\
\hline Lorazépam & $\begin{array}{c}0,260 \\
(0,010-0,100)\end{array}$ & 0,26 & 0,07 & 0,04 \\
\hline Lormétazépam & $\begin{array}{c}0,180 \\
(0,002-0,030)\end{array}$ & 0,39 & 0,24 & 0,15 \\
\hline
\end{tabular}




\section{Observation 2}

Madame D... lors de la consultation ne présente que des troubles cliniques et comportementaux mineurs. Ceux-ci sont interprétés par le praticien qui la voit comme les stigmates possibles d'une rechute de la toxicomanie à l'hérö̈ne. Cette hypothèse est infirmée par l'analyse toxicologique. Elle montre en revanche une conduite toxicophile aux benzodiazépines, des concentrations sanguines considérables en nordazépam, diazépam et lormétazépam, respectivement plus de 26 fois, 11 fois et 6 fois les valeurs thérapeutiques maximales usuelles. L'imprégnation capillaire excessive confirme l'exposition régulière et vraisemblablement très importante à ces molécules.

\section{Concernant les deux observations}

Les concentrations des benzodiazépines dans le sang, dans nos deux observations, sont comparables, voire supérieures à celles mesurées chez des sujets décédés d'intoxication aiguë par benzodiazépine seule comme l'indiquent les valeurs provenant d'une revue de la littérature mise à jour (4) et reportées dans le tableau II. $\mathrm{Ce}$ tableau comporte également des concentrations mesurées chez des sujets toxicomanes décédés d'intoxication aiguë par benzodiazépine. Les cas tirés de notre expérience médico-légale confortent la revue de la littérature. Ils montrent que la tolérance peut, avec ces molécules, se développer à un niveau extraordinairement élevé qui doit rendre extrêmement prudent quant à l'interprétation des résultats tant en toxicologie clinique qu'en toxicologie médico-légale.
En ce qui concerne l'analyse des benzodiazépines dans les cheveux, les données de la littérature rapportés par Cirimèle (11) sont regroupées dans le tableau III. Les valeurs obtenues dans les cheveux de nos deux malades sont comparables voire supérieures à celles de sujets pharmacodépendants. En ce qui concerne le lormétazépam, la concentration mesurée dans notre second cas est inférieure aux valeurs rapportées par Yegles (12), mais il existe vraisemblablement un biais méthodologique, ce dernier ayant réalisé les dosages par CG/SM en mode impact électronique. Chez nos deux malades, l'analyse des cheveux a permis de valider la conduite toxicophile aux benzodiazépines, déjà suggérée par l'important décalage entre l'état clinique constaté et les concentrations sanguines mesurées.

\section{Conclusion}

Ces deux patients illustrent parfaitement l'extraordinaire tolérance aux benzodiazépines susceptible de s'installer chez des sujets présentant manifestement une conduite toxicophile à ces médicaments. L'intérêt de ces observations, documentées par des dosages sanguins, permet dans les deux cas, grâce à l'analyse des cheveux d'apporter des informations pertinentes contribuant au diagnostic de toxicomanie aux benzodiazépines. L'ensemble de ces données a éclairé de manière objective l'équipe soignante, pour une prise en charge optimale de ces malades et a permis de définir clairement la stratégie médicale la mieux adaptée. Il n'en demeure pas moins que le problème du contrôle des prescriptions et de la délivrance de ces médicaments reste posé, ne serait-ce que dans l'intérêt des malades.

Tableau II : Concentrations sanguines dans les intoxications mortelles par benzodiazépines seules comparées à ces deux observations.

\begin{tabular}{|c|c|c|c|}
\hline SANG & n & Concentrations (mg/) & Auteurs \\
\hline Nordazépam & 3 & $4,4-4,2$ et $6,0(+$ oxaz 0,96$)$ & Goullé (5) \\
\hline $\begin{array}{l}\text { observation } 1 \\
\text { observation } 2\end{array}$ & & $\begin{array}{c}54 \\
31,7\end{array}$ & \\
\hline Diazépam & $\begin{array}{l}1 \\
2 \\
3 \\
5\end{array}$ & $\begin{array}{c}30(+ \text { alcool } 0,35 \mathrm{~g} / \mathrm{l}) \\
5 \text { et } 19 \\
\text { moy } 4,8 \\
\operatorname{moy} 5,2\end{array}$ & $\begin{array}{l}\text { Cardauns (6) } \\
\text { Finkle (7) } \\
\text { Dinovo (8) } \\
\text { Dinovo (8) }\end{array}$ \\
\hline observation 2 & & 8,2 & \\
\hline Oxazépam & $\begin{array}{l}4 \\
2 \\
\end{array}$ & $\begin{array}{c}0,4 \text { à } 4,6(\operatorname{moy} 2,6) \\
4,4 \text { et } 6,1\end{array}$ & $\begin{array}{c}\text { Drummer (9) } \\
\text { Druid (10) }\end{array}$ \\
\hline $\begin{array}{l}\text { observation } 1 \\
\text { observation } 2\end{array}$ & & $\begin{array}{l}6,5 \\
3,2\end{array}$ & \\
\hline $\mathrm{n}=$ nombre de cas & & & \\
\hline
\end{tabular}


Tableau III : Benzodiazépines dans les cheveux : concentrations publiées comparées à celles de ces deux observations.

\begin{tabular}{|c|c|c|c|c|}
\hline $\begin{array}{c}\text { CHEVEUX } \\
(\mathrm{n}=\text { nombre de cas })\end{array}$ & \multirow{2}{*}{$\begin{array}{c}\begin{array}{c}\text { Concentration } \\
\text { ng/mg }\end{array} \\
0,25 \text { à } 18,9 \\
(\text { moy } 4,16)\end{array}$} & \multirow{2}{*}{$\begin{array}{c}\text { CG/SM } \\
\mathrm{ICN}\end{array}$} & \multirow{2}{*}{$\begin{array}{c}\text { Traitement } \\
\text { Toxicomanes }\end{array}$} & \multirow{2}{*}{\begin{tabular}{|c|} 
Auteur \\
Kintz (3)
\end{tabular}} \\
\hline Nordazépam & & & & \\
\hline $\mathrm{n}=20$ & 0,13 à 1,83 & IE & $\begin{array}{c}\text { Toxicomanes } \\
\text { décédés* }\end{array}$ & Yegles (12) \\
\hline $\begin{array}{l}\text { observation } 1 \\
\text { observation } 2\end{array}$ & $\begin{array}{l}6,23 \\
5,77\end{array}$ & $\begin{array}{l}\text { ICN } \\
\text { ICN }\end{array}$ & & \\
\hline Oxazépam & $\begin{array}{l}0,11 \text { à } 0,50 \\
(\operatorname{moy} 0,28)\end{array}$ & $\mathrm{ICN}$ & Toxicomanes & Kintz (3) \\
\hline $\begin{array}{l}\text { observation } 1 \\
\text { observation } 2\end{array}$ & $\begin{array}{l}0,95 \\
0,67\end{array}$ & $\begin{array}{l}\text { ICN } \\
\text { ICN }\end{array}$ & & \\
\hline Diazépam & 0,01 à 2,21 & $\mathrm{IE}$ & $\begin{array}{c}\text { Toxicomanes } \\
\text { décédés* }\end{array}$ & Yegles (12) \\
\hline observation 2 & 3,02 & ICN & & \\
\hline Lorazépam & $\begin{array}{ll}0-2 \mathrm{~cm} & 0,049 \\
2-5 \mathrm{~cm} & 0,040 \\
5-9 \mathrm{~cm} & 0,040 \\
9-16 \mathrm{~cm} & 0,031\end{array}$ & $\mathrm{ICN}$ & $\begin{array}{c}\text { Temesta }^{\circledR} 1 \mathrm{mg} \\
\text { en grande } \\
\text { quantité pour } \\
\text { anxiété persistante }\end{array}$ & Cirimele (13) \\
\hline observation 2 & 0,260 & ICN & & \\
\hline Lormétazépam $\quad n=3$ & 4,07 à 29,05 & IE & $\begin{array}{l}\text { Toxicomanes } \\
\text { décédés* }\end{array}$ & Yegles (12) \\
\hline observation 2 & 0,39 & ICN & & \\
\hline \multicolumn{5}{|c|}{$\begin{array}{l}\text { IE = Impact Electronique }- \text { ICN = Ionisation Chimique Négative } \\
\text { * Les concentrations mesurées chez des toxicomanes décédés correspondent à la période précédant de plusieurs } \\
\text { semaines ou mois le décès. }\end{array}$} \\
\hline
\end{tabular}

\section{Références}

1. Flesch F., Jaeger A., Harlay M.L., Latrech B., Sauder P. Intoxications par benzodiazépines : analyse de 4078 cas. 9ème congrès de la Société Française de Toxicologie Analytique. La Clusaz, 12-16 mars 2001.

2. Nisse P., Deveaux M., Linke J.C., Ghysel M.H., Peucelle D., Mathieu-Nolf $M$. Intoxications par des benzodiazépines : revue des 157 décès survenus dans le nord de la France au cours des 5 dernières années. 9ème congrès de la Société Française de Toxicologie Analytique. La Clusaz, 12-16 mars 2001.

3. Kintz P., Cirimèle V., Vayssette F., Mangin P. Hair analysis for nordazepam and oxazepam by gas chromatography-negative-ion chemical ionization mass spectrometry. J. Chromatogr. B. $1996 ; 677: 241-44$.

4. Richard N., Ghysel M.H., Savart M. Intoxications mortelles par les benzodiazépines : applications médicolégales. Toxicorama. $1996 ; 8: 17-22$.

5. Goullé J.P., Lacroix C., Anagnostides J.G., Droy J.M., Gharios G., Proust B., Roumajon A., Gelis R., Ragot A., Vaz E. Les morts toxiques en Haute Normandie en 1994 et 1995. Journée de la Société Française de Toxicologique Clinique et de la Société Française de Toxicologie Analytique. Paris, 10 mars 1997.
6. Cardauns H., Iffland R. Ueber eine tödliche Diazepam (Valium ${ }^{\circledR}$ ) Vergiftung bei einem drogenabhängigen Jugendlichen. Arch. Toxikol. 1973 ; 31 : 147-51.

7. Finkle B.S., Mc Closkey K.L., Goodman L.S. Diazepam and drug associated deaths. JAMA. $1979 ; 242: 429-34$.

8. Dinovo E.C., Gottschalk L.A., Mc Guire F.L. Analysis of results of toxicological examinations performed by coroner's or medical examiner's laboratories in 2000 drug involved in nine major U.S. cities. Clin Chem. 1976 ; $22: 847-50$.

9. Drummer O.H. Death associated with Benzodiazepines. Proeceedings of the 1994 joint TIAFT/SOFT international meeting, $1994: 19-24$.

10.Druid H., Holmgren P. A complication of fatal and control concentrations of drugs in post-mortem femoral blood. J. Forensic Sci. 1997 ; 42 : 79-87.

11.Cirimèle V., Kintz P. Identification des benzodiazépines dans les cheveux : revue de la littérature. Toxicorama. $1996 ; 8: 75-9$.

12. Yegles M., Mersch F., Wennig R. Detection of benzodiazepines and other psychotic drugs in human hair by GC/MS. Forensic Sci. Int. 1997 ; 84 : 211-8.

13.Cirimele V., Kintz P., Mangin P. Detection and quantification of lorazepam in human hair by GC-MS/NCI in a case of traffic accident. Int. J. Legal Med. $1996 ; 108$ : 265-7. 